\title{
Efficacy of a New Preparation Regimen with Mosapride and Low Volume of Water Intake for Endoscopy Preparation in Postgastrectomy Patients
}

\author{
Tae Oh Kim ${ }^{*}$, Choi Joon Hyuk ${ }^{1}$, NaeYun Heo ${ }^{1}$, Jongha Park ${ }^{1}$, Jongha Park ${ }^{1}$, Seung Ha Park ${ }^{1}$, Sung \\ Yeon Yang ${ }^{1}$, Young Soo Moon ${ }^{1}$, Sung Jin $\mathrm{Oh}^{2}$, Byoung Jo Suh ${ }^{2}$
}

${ }^{1}$ Department of Internal Medicine, Haeundae Paik Hospital, Inje University College of Medicine, Busan

${ }^{2}$ Department of Surgery, Haeundae Paik Hospital, Inje University College of Medicine, Busan

*Corresponding Author: Tae Oh Kim, M.D. Ph.D., Department of Internal Medicine, Haeundae Paik Hospital, Inje University College of Medicine, 1435 Jwa-dong, Haeundae-gu, Busan 612-030, Republic of Korea, Tel: +82.51-797-0221; Fax: +82.51-7970298; E-mail: kto0440@paik.ac.kr

\begin{abstract}
Aim: Food retention is a frequent problem in patients who have previously undergone subtotal gastrectomy, which often makes endoscopy unsuccessful. This study evaluated the efficacy and patient tolerance of a new preparation regimen for upper gastrointestinal (UGI) endoscopy in patients who have undergone subtotal gastrectomy. Methods: A total of 135 patients with gastric cancer who underwent subtotal gastrectomy were enrolled for the study. They were divided into 3 groups: group A (40 patients) was used with mosapride with low-volume water intake; group B (40 patients) was used with large-volume water intake; group C (55 patients) underwent prolonged fasting before UGI endoscopy. The degree of food residue in the remnant stomach was classified according to endoscopic scoring system by blinded examiners. The tolerance of the new endoscopy preparation was assessed via questionnaires.

Results: The mean residual food scores were lower in group A than in group C $(0.35 \pm$ $0.58 v s .0 .87 \pm 0.84, \mathrm{p}=0.002)$. The group with the highest value of negative food residue was group A (70.0\%), followed by group B (65.0\%), and finally group C (40.0\%). Tolerance to the preparation was higher, although not significantly different, in group B compared to group A ( $80 \%$ vs. $60 \%, \mathrm{p}=0.149)$.

Conclusions: A combination of mosapride with low-volume water intake could be a more appropriate preparation for endoscopy in patients who have undergone subtotal gastrectomy.
\end{abstract}

Received Date: February 03, 2017 Accepted Date: February 28, 2017 Published Date: March 10, 2017

Citation: Tae Oh, Kim., et al. Efficacy of a New Preparation Regimen with Mosapride and Low Volume of Water Intake for Endoscopy Preparation in Postgastrectomy Patients. (2017) J Gastrointest Disord Liver Func 3(1): 89- 93.

\section{DOI: $10.15436 / 2471-0601.17 .1332$}

Keywords: Stomach neoplasm; Subtotal gastrectomy; Endoscopy; Mosapride; Water; Food residue

\section{Introduction}

Gastric cancer is the leading cancer type and the second most common cause of cancer deaths in Korea ${ }^{[1]}$. It has been reported that the incidence of gastric cancer in the remnant stomach is increasing owing to prolonged survival of gastric cancer patients $^{[2]}$. Early detection and appropriate management of these patients can result in a more favorable outcome ${ }^{[3]}$. Therefore, periodic endoscopic examinations are important for patients who have undergone a subtotal gastrectomy. During endoscopic examination, food residue is frequently found in the remnant stom- ach in $18 \%$ to $42 \%$ of patients, which occasionally prevents a clear observation of the stomach, and therefore interferes with early detection of recurrent carcinomas. Moreover, food residue may also be a risk factor for pulmonary aspiration of stomach contents ${ }^{[4,5]}$. However, to date, there is limited data regarding the effects of different preparation regimens for endoscopy in postgastrectomy patients. One recent study showed that the intake of a large quantity of water (1L) in a short time was effective and convenient for endoscopy preparation in patients who presented food residue ${ }^{[6]}$. However, ingesting a large volume of water is not well tolerated by the patients. In a previous report, oral 
mosapride, a gastroprokinetic agent that is used for the treatment of acid reflux and irritable bowel symptoms, accelerated gastric emptying and enhanced food intake after gastrointestinal surgery ${ }^{[7]}$. Therefore, we hypothesized that using mosapride together with low-volume water intake would be more efficient in preventing residual food and would allow better preparation for endoscopy. Our goal was to evaluate the degree of food retention in the remnant stomach after taking mosapride with a low volume of water and comparing this regimen with both the routine fasting preparation method and the large-volume water-intake method.

\section{Methods}

\section{Study design}

A prospective, single-center, randomized, blind study was carried out to evaluate the degree of food residue in the remnant stomach as well as the level of patient tolerance to 3 types of preparations for upper GI endoscopy. Between December 2014 and September 2015, at the gastroenterology clinic in Haeundae Paik Hospital, Busan, Korea, upper endoscopy examinations were performed in 135 patients who had previously undergone subtotal gastrectomy for gastric cancer. The study protocol was approved by the institutional review board of Inje University Haeundae Paik Hospital, and patients provided written informed consent before enrolling in the study.

The following exclusion criteria were established. Patients with recurrences of advanced gastric cancer or anastomotic stricture, severe underlying diseases that required therapy (renal failure or congestive heart failure, or uncontrolled diabetes mellitus were excluded. During the study, 3 patients who did not follow the specified protocol for endoscopy preparation, 1 who had anastomotic stricture, and 1 who had a combined investigation with colonoscopy $(n=1)$ were excluded. The data on the primary outcome measures were complete for all 135 patients and were included in the final analysis (Figure 1). The questionnaire administered to the patients included an evaluation of the clinical factors before endoscopy. Medical records were reviewed retrospectively to assess the clinicopathological or surgical features of the patients.

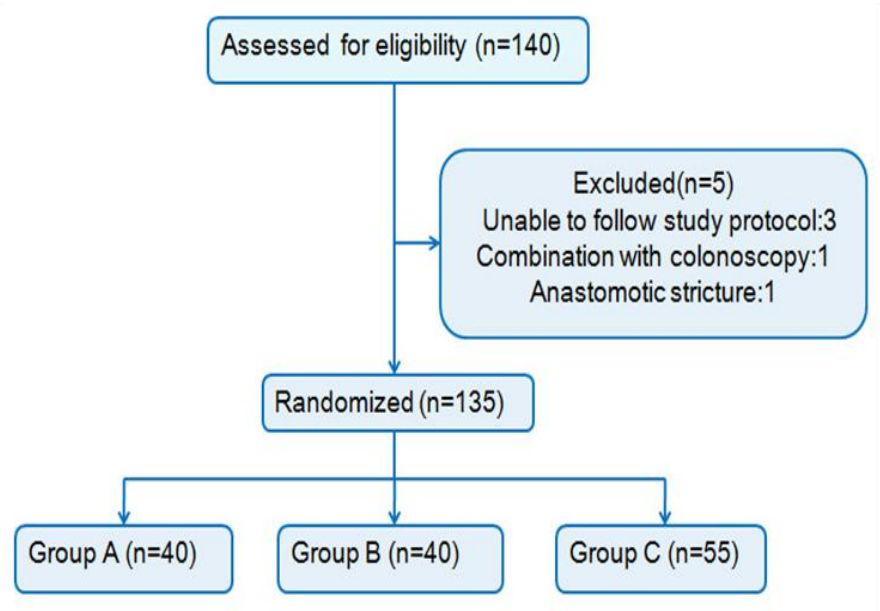

Group A: Mosapride with low volume water group

Group B: Large volume water group

Group C: Prolonged fasting group (Control)

\section{Protocol for preparation}

The efficacy of the 3 methods of patient preparation for endoscopy was used as the primary endpoint of the current study. The regimen for group A (mosapride with low-volume water intake) was used for 40 examinations. Patients had a soft diet dinner, took $10 \mathrm{mg}$ of mosapride at 19:00, drank $200 \mathrm{~mL}$ of water at intervals of 10minutes for a total of 0.4L between 19:00 and 19:30, and then fasted from 21:00 until the next day. On the day of the endoscopy, the patients took $10 \mathrm{mg}$ of mosapride at 07:00. The regimen for group B (large-volume water intake) was used for 40 examinations. Patients had a soft diet dinner, drank $200 \mathrm{~mL}$ of water at intervals of 10 minutes for a total of 1L between 19:00 and 20:00 and then fasted from 21:00 until the next day. The regimen for group $\mathrm{C}$ (prolonged fasting, control group) was used for 55 examinations. This group followed the conventional preparation: the patients had a soft diet dinner the day before endoscopy and fasted until the endoscopy. All endoscopic examinations were performed between 09:00 and 12:00 by 2 experienced endoscopists, blinded to the allocated treatments and the randomized grouping.

\section{Degree of food residue}

The amount of residual food was classified into 4 grades according to the endoscopic scoring system ${ }^{[4]}$. Grade 0 was defined as no food residue in the stomach; grade 1, small amount of food residue; grade 2, moderate amount of food residue that allowed the observation only of the side of the posterior wall and the lesser curvature of the stomach; and grade 3, large amount of food residue due to which endoscopic observation was impossible. The results of examinations with absence of food residue, corresponding to grade 0 , were defined as "negative" food residue, whereas those with presence of food residue were defined as "positive" food residue and classified as grade 1, 2 and 3 . The endoscopic scores were evaluated by 1 investigator and 1 independent assessor. If there was any disagreement on the scoring between them, the lower grade was used. To minimize inter-observer variation, frequent discussion meetings were held to provide each participating endoscopist with standard instructions for scoring.

\section{Tolerability of endoscopic preparation regimens}

As the secondary endpoint of the study, we assessed the tolerability of the preparation regimens via a questionnaire. Immediately before the procedure, patients completed a questionnaire regarding their preparation experience with the help of a research nurse, who was blinded to group allocation. Patients were asked about the tolerability of the preparations. The level of tolerability was graded according to 3 categories: "good", "fair", or "poor".

\section{Statistical analysis}

All statistical analyses were performed using the SPSS program, version 18.0 (SPSS Inc, Chicago, IL, USA). Continuous variables such as mean food residue scores were compared with an ANOVA test. The chi-squared test or Fisher's exact test were used to assess the relationship between categorical variables such as the grade of food residue and presence or absence of a specific adverse event. Results were considered statistically significant when the $\mathrm{p}$-value was $\leq 0.05$.

Figure 1: Results of this study strategy 


\section{Results}

\section{Patient characteristics}

A total of 135 patients who were scheduled for endoscopy after subtotal gastrectomy were included in the final analysis. The age of the patients ranged from 32 to 80 years, with a mean of $59.5 \pm 10.7$ years. There were no significant differences in the clinical factors among the patients in the preparation groups (Table 1).

Table 1: Comparisons of clinicopathologic factors among the 3 preparation groups.

\begin{tabular}{|c|c|c|c|c|}
\hline & $\begin{array}{l}\text { Group A } \\
(\mathrm{n}=\mathbf{4 0})\end{array}$ & $\begin{array}{l}\text { Group B } \\
(n=40)\end{array}$ & $\begin{array}{l}\text { Group C } \\
(\mathrm{n}=\mathbf{5 5})\end{array}$ & p-value \\
\hline Age & $58.5 \pm 10.9$ & $58.3 \pm 8.1$ & $61.1 \pm 12.0$ & 0.353 \\
\hline $\operatorname{Sex}(M / F)$ & $20 / 20$ & $26 / 14$ & $37 / 18$ & 0.200 \\
\hline \multicolumn{5}{|c|}{ History of abdominal surgery } \\
\hline Positive & 8 & 10 & 9 & 0.165 \\
\hline Negative & 32 & 30 & 42 & \\
\hline \multicolumn{5}{|c|}{ Depth of tumor invasion } \\
\hline Early gastric cancer & 36 & 36 & 43 & 0.165 \\
\hline $\begin{array}{l}\text { Advanced gastric } \\
\text { cancer }\end{array}$ & 4 & 4 & 12 & \\
\hline LADG* & 8 & 18 & 17 & 0.055 \\
\hline \multicolumn{5}{|l|}{ Reconstruction } \\
\hline Billroth I & 12 & 14 & 15 & 0.720 \\
\hline Billroth II & 28 & 26 & 40 & \\
\hline \multicolumn{5}{|c|}{ Gastrectomy to endoscopy interval } \\
\hline$<1$ years & 14 & 16 & 23 & 0.132 \\
\hline $1-2$ years & 2 & 8 & 4 & \\
\hline$>2$ years & 24 & 16 & 28 & \\
\hline
\end{tabular}

Group A: Mosapride with low-volume water-intake group Group B: Large-volume water-intake group

Group C: Prolonged fasting group (control group)

LADG*, laparoscopy-assisted distal gastrectomy

Table 2: Relationship between preparation methods and food residue.

\begin{tabular}{|l|l|l|l|l|l|}
\hline & $\begin{array}{l}\text { Group A } \\
(\mathbf{n}=\mathbf{4 0})\end{array}$ & $\begin{array}{l}\text { Group B } \\
(\mathbf{n}=\mathbf{4 0})\end{array}$ & $\begin{array}{l}\text { Group C } \\
(\mathbf{n}=\mathbf{5 5})\end{array}$ & p-value & $\begin{array}{l}\text { Post hoc } \\
\text { analysis }\end{array}$ \\
\hline Food residue score \\
\hline mean \pm SD & $0.35 \pm 0.58$ & $0.50 \pm 0.75$ & $0.87 \pm 0.84$ & 0.002 & $\begin{array}{l}\mathrm{A}<\mathrm{B}(\mathrm{p}=0.686) \\
\mathrm{B}<\mathrm{C}(\mathrm{p}=0.075) \\
\mathrm{C}>\mathrm{A}(\mathrm{p}=0.002)\end{array}$ \\
\hline Grade 0 & $28(70.0)$ & $26(65.0)$ & $22(40.0)$ & $0.022^{*}$ & \\
\hline Grade 1 & $10(25.0)$ & $8(20.0)$ & $19(34.5)$ & & \\
\hline Grade 2 & $2(5.0)$ & $6(15.0)$ & $13(23.6)$ & & \\
\hline Grade 3 & $0(0.0)$ & $0(0.0)$ & $1(1.8)$ & & \\
\hline Food residue & & & \\
\hline Negative & $28(70.0)$ & $26(65.0)$ & $22(40.0)$ & $0.006^{*}$ & \\
\hline Positive & $12(30.0)$ & $14(35.0)$ & $33(60.0)$ & & \\
\hline
\end{tabular}

Values shown are number (\%) unless otherwise indicated.

Group A: Mosapride with low-volume water-intake group

Group B: Large-volume water-intake group

Group C: Prolonged fasting group (control group)

*Fisher's exact test

\section{Assessment of food residue}

The differences in mean scores of food residue among the 3 groups were significantly different $(p=0.002)$. Post hoc analysis results showed that group A had lower mean food residue than that of group $\mathrm{C}$. In addition, the classification of presence of food residue among the 3 groups were also significantly different $(p=0.022)$. The group with the highest value of negative food residue was group A $(70.0 \%)$, followed by group B $(65.0 \%)$, and finally group C $(40.0 \%)(p=0.006$; Table 2$)$.

\section{Tolerability and adverse experiences}

Tolerability of the preparation regimens was compared only between the mosapride group (A) and large-volume water-intake group (B). The convenience of the endoscopic preparation regimen was graded as "good" by a higher percentage of patients in group B $(80 \%)$ than in group A $(60 \%)(p=0.149$; Figure 2). No serious adverse events were encountered during or after the endoscopy preparation process.

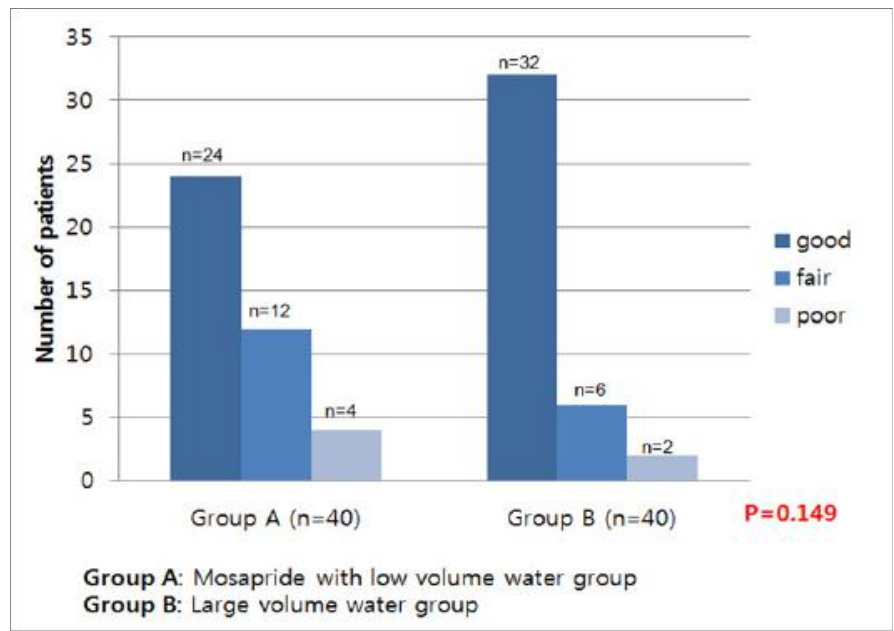

Figure 2: Tolerability scores of the preparation methods. The dark blue bar represents patients that graded the preparation as "good," the blue bar as "fair," and the light blue bar as "poor."

\section{Discussion}

Distal subtotal gastrectomy is the standard operative procedure for gastric cancer ${ }^{[6,8,9]}$. During the follow-up of subtotal gastrectomy-treated patients, we identified several patients with a large amount of food residue. Food residue in the remnant stomach interferes with close endoscopic observation, and therefore early detection of recurrent carcinoma, and may increase the risk of pulmonary aspiration during upper endoscopy ${ }^{[5,6]}$. Food retention may be a consequence of overeating, decreased gastric motility, or a mechanical obstruction due to gastroenterostomy, such as stenosis or stricture ${ }^{[3]}$. The effective preparation method for endoscopy is important to detect early lesions or perform endoscopic resection of polyps or dysplastic lesions in the remnant stomach during follow-up endoscopy. Few studies have analyzed the effectiveness of the preparation methods for endoscopic examination in reducing the amount of food residue in patients after distal gastrectomy. It has been shown that preparation regimens using a hypermotility drug, such as domperidone, were not effective, whereas dietary controls, such as fasting or a liquid diet could reduce the amount of food residue ${ }^{[5]}$. Indeed, a recent study showed that drinking a large amount of water (up to 
1L) in a short time before endoscopy was effective in reducing food residue in patients who had distal gastrectomy ${ }^{\left[{ }^{[6]}\right.}$. However, although this regimen could reduce food residue more effectively than conventional prolonged fasting, it was not convenient for the patient as the excessive water created mild discomfort. Recently, studies in which various prokinetic agents were administered to the patients before endoscopic examination have shown promising results ${ }^{[10-12]}$. One of these agents, mosapride citrate, accelerated gastric emptying and increased the completion rate of small bowel examination in patients undergoing capsule endoscopy ${ }^{[13]}$. Mosapride is a 5-HT4 receptor agonist ${ }^{[14]}$. This drug does not antagonize dopamine D2 receptors, but selectively acts on 5-HT4 receptors, thus enhancing gastrointestinal motility through the acceleration of acetylcholine release from the nerves in the gastrointestinal tract ${ }^{[15]}$. These effects, in turn, accelerate gastric emptying. Wei et $\mathrm{a}^{\left[{ }^{[13]}\right.}$. assessed the effect of oral mosapride on gastrointestinal transit time and diagnostic yield of capsule endoscopy and found that $10 \mathrm{mg}$ of mosapride was well tolerated and enhanced gastric emptying. We hypothesized that mosapride associated with low-volume water intake could reduce food residue in the stomach, and therefore could represent a more convenient method for endoscopic preparation in patients who have undergone distal gastrectomy. Analysis of the primary endpoint of our study showed that oral administration of mosapride with low-volume water intake was more efficient in reducing food retention compared to conventional prolonged fasting. The mean score for food residue was effectively lower compared to the existing method. The negative food residue score decreased sequentially from the mosapride with low-volume water-intake group, to the large-volume water-intake group to the control group. However, in our study, the mean score in the large-volume water-intake group was similar to the score in the control group. This results in agreement with previous studies, which were designed to survey the high-risk groups of patients who failed the first endoscopy due to the presence of large amounts of food residue, although our study was not designed with this bias. The results of the tolerance scores to the preparation methods were different from what we hypothesized at the beginning of the study. A higher percentage of the patients of the large-volume water-intake group scored the preparation as "good" compared to the mosapride group, although the differences were not statistically significant. Similar results were obtained when we grouped "good" and "fair" in the same category as "easy preparation" and "poor" in the "difficult preparation" category. Although very promising, our study has some limitations that need to be taken into account. Several prokinetic agents are known to increase gastric emptying, but for some of them, the outcomes when used for endoscopy preparation were questionable. In this study, we used mosapride, but more studies are needed with different kinds of prokinetics in order to identify the most efficient ones and verify whether different kinds of prokinetics give similar results. Another limitation of this study is the absence of a standard endoscopic scoring system. There is no universally accepted standard scale to accurately quantify and compare grades of food residue. In most cases, investigators have used a variety of endoscopic score scales; therefore, comparisons between studies are difficult. A more uniform endoscopic scoring system would possibly lead to a more consistent classification of food retention. It is well known that there are several risk factors associated with food residue after gas- trectomy. Among these are the presence of underlying diseases, such as diabetes and hypothyroidism, the reconstruction method after distal gastrectomy, the diameter of the anastomotic site, the amount of time since the operation, and several others ${ }^{[3,5,15-17]}$. In our study, however, we did not assess the correlation between risk factors and food retention. Further studies will be needed to evaluate the relationship between risk factors and food retention in patients who have undergone subtotal gastrectomy. Taken together, our results showed some important differences in the amount of food residue in the remnant stomach with the 3 methods of preparation. Indeed, the combination of mosapride with water provides a more effective way to reduce food residue. Moreover, this regimen has the advantages of being practical and very easily prepared. In conclusion, treatment with oral mosapride and a low-volume water intake on the day before endoscopy can be recommended as a suitable preparation regimen for endoscopy in patients who have undergone subtotal gastrectomy.

\section{References}

1. Shin, A., Kim, J., Park, S. Gastric cancer epidemiology in Korea. (2011) J Gastric Cancer 11(3): S135-S140.

Pubmed | Crossref | Others

2. Takeno, S., Noguchi, T., Kimura, Y., et.al. Early and late gastric cancer arising in the remnant stomach after distal gastrectomy. (2006) Eur J Surg Oncol 32(10): S1191-S1194.

Pubmed | Crossref|Others

3. Jung, HJ., Lee, JH., Ryu, KW., et.al. The influence of reconstruction methods on food retention phenomenon in the remnant stomach after a subtotal gastrectomy. (2008) J Surg Oncol 98(1): S11-S14.

Pubmed | Crossref | Others

4. Kubo, M., Sasako, M., Gotoda, T., et.al. Endoscopic evaluation of the remnant stomach after gastrectomy: proposal for a new classification. (2002) Gastric Cancer 5(2): S83-S89.

Pubmed | Crossref| Others

5. Watanabe, H., Adachi, W., Koide, N., et.al. Food residue at endoscopy in patients who have previously undergone distal gastrectomy: risk factors and patient preparation. (2003) Endoscopy 35(5): S397-S401. Pubmed | Crossref| Others

6. Cho, SB., Yoon, KW., Park, SY., et al. Risk factors for food residue after distal gastrectomy and a new effective preparation for endoscopy: the water-intake method. (2009) Gut Liver 3(3): S186-S191.

Pubmed | Crossref|Others

7. Toyomasu, Y., Mochiki, E., Morita, H., et al. Mosapride citrate improves postoperative ileus of patients with colectomy. (2011) J Gastrointest Surg 15(8): S1361-S1367.

Pubmed |Crossref|Others

8. Nagano, H., Ohyama, S., Sakamoto, Y., et.al. The endoscopic evaluation of gastritis, gastric remnant residue, and the incidence of secondary cancer after pylorus-preserving and transverse gastrectomies. (2004) Gastric Cancer 7(1): S54-S59.

Pubmed | Crossref | Others

9. Folli, S., Dente, M., Dell'Amore, D., et.al. Early gastric cancer: prognostic factors in 223 patients. (1995) Br J Surg 82(7): S952-S956. Pubmed | Crossref | Others

10. Cho, YK., Choi, MG., Han, HW., et al. The effect of mosapride on esophageal motility and bolus transit in asymptomatic volunteers. (2006) J Clin Gastroenterol 40(4): S286-S292.

Pubmed | Crossref | Others

11. Markey, O., Shafat, A. Does domperidone, a D(2)-antagonist alter gastric emptying rates and appetite sensations in healthy adults with high-fat meal? A block-randomised, single-blind placebo-controlled study. (2011) Ir J Med Sci 181(2): S215-S219.

Pubmed | Others 
12. Masaoka, T., Tack, J. Gastroparesis: Current concepts and management. (2009) Gut Liver 3(3): S166-S173.

Pubmed|Crossref|Others

13. Wei, W., Ge, ZZ., Lu, H., et.al. Effect of mosapride on gastrointestinal transit time and diagnostic yield of capsule endoscopy. (2007) J Gastroenterol Hepatol 22(10): S1605-S1608.

Pubmed | Crossref | Others

14. Jung, IS., Kim, JH., Lee, HY., et.al. Endoscopic evaluation of gastric emptying and effect of mosapride citrate on gastric emptying. (2010) Yonsei Med J 51(1): S33-S38.

Pubmed|Crossref

15. Endo, J., Nomura, M., Morishita, S., et al. Influence of mosapride citrate on gastric motility and autonomic nervous function: evaluation by spectral analyses of heart rate and blood pressure variabilities, and by electrogastrography. (2002) J Gastroenterol 37(11): S888-S895.

Pubmed | Crossref| Others

16. Behrns, KE., Sarr, MG. Diagnosis and management of gastric emptying disorders. (1994) Adv Surg 27: S233-S255.

Pubmed

17. Le Blanc-Louvry, I., Savoye, G., Maillot, C., et.al. An impaired accommodation of the proximal stomach to a meal is associated with symptoms after distal gastrectomy. (2003) Am J Gastroenterol 98(12): S2642-S2647.

Pubmed | Crossref| Others 